Research Paper

\title{
Survival Contradiction Between Stage IIA and Stage IIIA Rectal Cancer: A Retrospective Study
}

\author{
Shaobo Mo ${ }^{1,2 *}$, Weixing Dai ${ }^{1,2 *}$, Wenqiang Xiang ${ }^{1,2 *}$, Ben Huang ${ }^{1,2}$, Yaqi Li1 ${ }^{1,2}$, Yang Feng ${ }^{1,2}$, Qingguo Li1 ${ }^{1,2 \varpi}$, \\ Guoxiang Cai ${ }^{1,2 \varpi}$ \\ 1. Department of Colorectal Surgery, Fudan University Shanghai Cancer Center, Shanghai 200032, China; \\ 2. Department of Oncology, Shanghai Medical College, Fudan University, Shanghai 200032, China. \\ *Shaobo Mo, Weixing Dai, and Wenqiang Xiang contributed equally to this work.
}

$\square$ Corresponding author: Guoxiang Cai, MD, PhD., Department of Colorectal Surgery, Fudan University Shanghai Cancer Center and Department of Oncology, Shanghai Medical College, Fudan University, 270 Dong'an Road, Shanghai, 200032, China. Tel: 86-18017312703; Fax: 86-021-54175590; E-mail: gxcaifuscc@163.com or Qingguo Li, MD, PhD., Department of Colorectal Surgery, Fudan University Shanghai Cancer Center and Department of Oncology, Shanghai Medical College, Fudan University, 270 Dong'an Road, Shanghai, 200032, China. Tel: 86-18918298120; Fax: 86-021-54175590; E-mail: oncosurgeonli@sohu.com

(c) Ivyspring International Publisher. This is an open access article distributed under the terms of the Creative Commons Attribution (CC BY-NC) license (https://creativecommons.org/licenses/by-nc/4.0/). See http://ivyspring.com/terms for full terms and conditions.

Received: 2017.10.13; Accepted: 2018.01.19; Published: 2018.04.06

\begin{abstract}
Background: When compared with patients harboring stage IIB and stage IIC disease, those with stage IIIA colorectal cancer have a better prognosis. We aimed to compare the cause-specific survival (CSS) of the patients with stage IIA rectal cancer with that of the patients with stage IIIA rectal cancer.

Methods: Data analyzed about patients with stage IIA and stage IIIA rectal cancer was from the US Surveillance, Epidemiology, and End Results (SEER) database. We then validated the results using data derived from Fudan University Shanghai Cancer Center (FUSCC).

Results: A total of 16,788 patients (13,551 staged IIA and 3,237 staged IIIA) were identified in SEER database. A multivariate analysis manifested that patients with stage IIIA disease were more likely to have a better CSS (HR 0.894, 95\% Cl 0.816-0.979, $\mathrm{p}=0.016$ ) compared with patients with stage IIA rectal cancer. In the subgroup of patients whose number of lymph nodes harvested $(\mathrm{LNH})<12$, multivariate analysis signified that patients with stage IIIA disease were more prone to have favorable CSS (HR 0.805, 95\% Cl 0.719-0.901, $p<0.001)$ compared with patients with stage IIA rectal cancer. In $\mathrm{LNH} \geq 12$ subgroup, the Kaplan-Meier analysis revealed no significant difference between patients experiencing stage IIA and IIIA rectal cancer $(p=0.618)$. Validation of data from FUSCC proved that patients with stage IIIA rectal cancer were more inclined to have better CSS (HR $0.407,95 \% \mathrm{Cl}$ $0.187-0.885, p=0.019$ ) in comparison to those with stage IIA rectal cancer. Specifically, in $\mathrm{LNH}<12$ subgroup, the survival outcomes of stage IIIA patients were significantly better than that of the stage IIA patients $(p=0.019)$, while there was no statistical significance between these two stages in the subgroup of patients with $\mathrm{LNH} \geq 12(\mathrm{p}=0.180)$.
\end{abstract}

Conclusions: Patients with stage IIA rectal cancer have poorer CSS than patients with stage IIIA rectal cancer, particularly when inadequate lymph nodes are harvested.

Key words: rectal cancer; survival; stage IIA; stage IIIA; lymph node retrieval

\section{Introduction}

Colorectal cancer (CRC) is the third most common carcinoma in the United States (US) in 2017[1] and the fifth commonly diagnosed malignancy in China in 2015[2], which still remains an important trigger of death and a major public health issue. According to heterogeneous oncological outcomes, stage III rectal cancer is subdivided into stage IIIA, stage IIIB and stage IIIC rectal cancer in the 
$7^{\text {th }}$ AJCC cancer staging manual[3]. Although the $7^{\text {th }}$ edition AJCC cancer staging system has some improvements over the $6^{\text {th }}$ edition $[4,5]$, a survival contradiction between stage IIIA and stage II colorectal cancer still exists in the current staging system[6-8]. Patients with stage IIIA colorectal cancer showed better prognosis than those with stage IIB and stage IIC disease in several studies[7-9]. However, few studies sought to compare patients with stage IIIA rectal cancer and those with stage IIA disease.

Lymph node status which can determine the postoperative treatments and follow-up plans has been identified as a crucial prognostic factor in colorectal cancer[10, 11]. Whether lymph node metastasis exists can distinguish stage III from stage II colorectal cancer. The AJCC and College of American Pathologists (CAP) recommended to examine at least 12 lymph nodes to adequately stage colorectal cancer patients[12-15]. An increased number of lymph nodes assessed correlates with better survival outcomes in patients with both stage II and stage III colorectal cancer. Peeples et al.[16] reported the 5-year survival rates were $52 \%$ (< 12 lymph nodes) and $63 \%(\geq 12$ lymph nodes) for patients with stage II colorectal cancer $(p<0.01)$. Some investigators[17] reported that patients with inadequate lymph nodes retrieved $(<12)$ had reduced overall survival $(p=0.001)$ and poorer disease-free survival $(p=0.001)$ compared with those with adequate lymph nodes retrieved $(\geq 12)$ in stage III colorectal cancer.

Using Surveillance, Epidemiology, and End Results (SEER) database, we aimed to compare the prognosis of patients with stage IIA with those harboring stage IIIA rectal cancer. The comparison between stage IIA and stage IIIA rectal cancer was then carried out at different levels of lymph nodes examined. Moreover, we further validated these pertinent issues in an inhouse set of patients undergoing stage IIA and stage IIIA rectal cancer from Fudan University Shanghai Cancer Center (FUSCC).

\section{Materials and Methods}

\section{Patient selection in SEER database}

The SEER program (http://seer.cancer.gov/) is sponsored by the National Cancer Institute. As a population-based cancer registry, it collects and publishes cancer incidence and survival data. The SEER program consists of 18 population-based cancer registries, by which around $28 \%$ of the US population is covered. Invasive rectal cancer cases from January 1988 to December 2011 were extracted from the SEER database (http://seer.cancer.gov, April 2013 release).
Inclusion criteria: adenocarcinoma, mucinous adenocarcinoma or signet-ring carcinoma of the rectum; lymph node status and known depth of invasion; AJCC stage IIA or stage IIIA; pathologically confirmed diagnosis; pathology specimen from surgical resection of rectal cancer; rectal cancer as the primary and only malignant tumor; and known survival time and cause of death. Those who received preoperative radiotherapy or had only local tumor excised were ruled out.

\section{Patient selection in the FUSCC cohort}

The prospective establishment of FUSCC rectal cancer dataset recorded the rectal cancer patients receiving treatment at FUSCC, Shanghai, China since January, 2006[18]. To prove the findings from the SEER set and to clarify related issues mentioned, we utilized rectal cancer data from FUSCC between January 2006 and December 2012. Consecutive patients who were histologically diagnosed to have a single primary tumor of AJCC stage IIA or IIIA rectal cancer, with accurate age at diagnosis, histological type, tumor size, pathological grade, and number of lymph node harvested ((LNH)) were included in our study. Patients who had received preoperative radiotherapy or been through only local resection were excluded from this study. Depending on the follow-up system of FUSCC, the clinical statistics center of this hospital offered the survival data. Patients who lived to the end of the follow-up were selected for analysis. All patients involved provided written informed consent. The study was approved by the Institutional Review Board of FUSCC.

\section{Outcome measures}

The following data were collected from the SEER database: sex, race, age at diagnosis (60 years was the cut-off in analyses), tumor size, pathological grade, histological type, primary site, number of primaries, number of lymph nodes harvested, number of positive lymph nodes, depth of local tumor invasion, AJCC TNM stage, radiation sequence with surgery, follow-up time, and SEER cause-specific death classification. All patients were restaged according to the latest AJCC Cancer Staging Manual, in which stage IIA was defined as T3N0M0 and stage IIIA was defined as T1-2N1M0 or T1N2aM0. The primary end-point of our study was cause-specific survival (CSS), namely, the time of diagnosis to the time when rectal cancer-specific death occurs. Deaths due to rectal cancer were regarded as events, and deaths from other causes or being alive at the end of follow-up time were censored. 
Table 1. Demographics of patients with stage IIA and stage IIIA rectal cancer in SEER and FUSCC cohorts [N (\%)]

\begin{tabular}{|c|c|c|c|c|c|c|}
\hline \multirow[t]{3}{*}{ Characteristics } & \multicolumn{3}{|c|}{ SEER Cohort } & \multicolumn{3}{|c|}{ FUSCC Cohort } \\
\hline & Total & IIA & IIIA & Total & IIA & IIIA \\
\hline & $(\mathrm{N}=16788)$ & $(\mathrm{N}=13551)$ & $(\mathrm{N}=3237)$ & $(\mathrm{N}=585)$ & $(\mathrm{N}=482)$ & $(\mathrm{N}=103)$ \\
\hline Median follow-up (mos) & 61 & 60 & 67 & 55 & 54 & 58 \\
\hline Mean tumor size $(\mathrm{cm})$ & 4.7 & 4.9 & 3.5 & 4.17 & 4.38 & 3.17 \\
\hline \multicolumn{7}{|l|}{ Sex } \\
\hline Male & $9444(56.3)$ & $7644(56.4)$ & $1800(55.6)$ & $360(61.5)$ & $312(64.7)$ & $48(46.6)$ \\
\hline Female & $7344(43.7)$ & $5907(43.6)$ & $1437(44.4)$ & $225(38.5)$ & $170(35.3)$ & $55(53.4)$ \\
\hline \multicolumn{7}{|l|}{ Age at diagnosis (yr) } \\
\hline$\leq 60$ & $5472(32.6)$ & $4133(30.5)$ & $1339(41.4)$ & $360(61.5)$ & $291(60.4)$ & $69(67.0)$ \\
\hline$>60$ & $11316(67.4)$ & $9418(69.5)$ & $1898(58.6)$ & $225(38.5)$ & 191(39.6) & $34(33.0)$ \\
\hline \multicolumn{7}{|l|}{ Race } \\
\hline White & $14005(83.4)$ & 11332(83.6) & $2673(82.6)$ & / & / & / \\
\hline Black & $1251(7.5)$ & $1008(7.4)$ & $243(7.5)$ & / & / & / \\
\hline Othera & $1532(9.1)$ & $1211(9.0)$ & $321(9.9)$ & / & / & / \\
\hline \multicolumn{7}{|l|}{ Histological Type } \\
\hline Adenocarcinoma & $15587(92.9)$ & $12560(92.7)$ & $3027(93.5)$ & $531(90.8)$ & $436(90.5)$ & 95(92.2) \\
\hline Mucinous adenocarcinoma & $1149(6.8)$ & $955(7.0)$ & $194(6.0)$ & $51(8.7)$ & $44(9.1)$ & $7(6.8)$ \\
\hline Signet-ring cell carcinoma & $52(0.3)$ & $36(0.3)$ & $16(0.5)$ & $3(0.5)$ & $2(0.4)$ & $1(1.0)$ \\
\hline \multicolumn{7}{|l|}{ Pathological grade } \\
\hline Well & $1280(7.6)$ & $1043(7.7)$ & $237(7.3)$ & $39(6.7)$ & $27(5.6)$ & $12(11.7)$ \\
\hline Moderate & $13345(79.5)$ & $10878(80.3)$ & $2467(76.2)$ & $529(90.4)$ & $441(91.5)$ & $88(85.4)$ \\
\hline Poor & $2084(12.4)$ & $1574(11.6)$ & $510(15.8)$ & $14(2.4)$ & $12(2.5)$ & $2(1.9)$ \\
\hline Undifferentiated & $79(0.5)$ & $56(0.4)$ & $23(0.7)$ & $3(0.5)$ & $2(0.4)$ & $1(1.0)$ \\
\hline \multicolumn{7}{|l|}{ LNH } \\
\hline$<12$ & $9230(55.0)$ & $7402(54.6)$ & $1828(56.5)$ & $128(21.9)$ & $99(20.5)$ & $29(28.2)$ \\
\hline$\geq 12$ & $7558(45.0)$ & $6149(45.4)$ & $1409(43.5)$ & $457(78.1)$ & $383(79.5)$ & $74(71.8)$ \\
\hline
\end{tabular}

$\mathrm{LNH}=$ number of lymph nodes harvested

a Includes Native American, Asian, Pacific Islander and Unknown

\section{Statistical analysis}

Clinicopathological data based on stage IIA and stage IIIA rectal cancer were summarized via cross-tabulation. The chi-squared tests were used to compare the distributions. Survival curves were drawn using Kaplan-Meier analysis, and the log-rank test was applied to figure out differences. Multivariate Cox proportional hazards models were used to analyze prognostic risk factors. In multivariate analysis, tumor size was analyzed as a continuous variable. All computed p-values were two-sided, and $\mathrm{p}<0.05$ was regarded as statistically significant. SPSS 20.0 was applied in all statistical analyses.

\section{Results}

\section{SEER database patient characteristics}

In the final analysis, there were a total of 16,788 patients (13,551 staged IIA and 3,237 staged IIIA), $23.8 \%$ of whom died from rectal cancer. Patient demographics and pathologic features are shown in Table 1. The median follow-up time was 61 months (interquartile range, 26-104 months). The study sample comprised 9,444 (56.3\%) males and 7,344 $(43.7 \%)$ females. The patients were mainly Caucasian (83.4\%), with African American (7.5\%) following. Histological types encompass adenocarcinoma $(92.9 \%)$, mucinous adenocarcinoma $(6.8 \%)$, and signet-ring cell carcinoma (0.3\%). When it comes to age at diagnosis $(p<0.001)$, histological type $(p=0.012)$ and pathological grade $(\mathrm{p}<0.001)$, there were significant differences between patients with stage IIA and stage IIIA rectal cancer.

\section{Survival paradox between stage IIA and stage IIIA rectal cancer in SEER database}

The Kaplan-Meier analysis showed that stage IIA patients had worse CSS than stage IIIA patients $(p<0.001$, Fig. 1A); the 5-year CSS of patients with stage IIA and stage IIIA rectal cancer were $77.1 \%$ and $81.6 \%$, respectively. Univariate analysis of the entire sample indicated that sex $(\mathrm{p}=0.005)$, age at diagnosis $(\mathrm{p}<0.001)$, race $(\mathrm{p}<0.001)$, histological type $(\mathrm{p}<0.001)$, pathological grade $(p<0.001)$, number of LNH $(\mathrm{p}<0.001)$, and tumor stage $(\mathrm{p}<0.001)$ were risk factors for CSS (Table 2). The following independent prognostic factors were identified by multivariate analysis: sex $(p<0.001)$, age at diagnosis $(p<0.001)$, race $(p<0.001)$, histological type $(\mathrm{p}<0.001)$, tumor size $(p<0.001)$, pathological grade $(p<0.001), \quad$ LNH $(p<0.001)$, and tumor stage $(p=0.016)$ (Table 3). Compared with stage IIA patients, stage IIIA patients had better CSS (HR 0.894, 95\% CI 0.816-0.979, $\mathrm{p}=0.016$ ).

In view of the recommendation from the AJCC and CAP that at least 12 lymph nodes of rectal cancer patients should be evaluated, we divided the study cohort into subgroups of patients with $\mathrm{LNH} \geq 12$ and LNH $<12$. In $\mathrm{LNH} \geq 12$ subgroup, The Kaplan-Meier analysis revealed no statistical significance between patients with stage IIA and IIIA rectal cancer $(p=0.618$, Fig. 1B); the 5-year CSS of patients with stage IIA and 

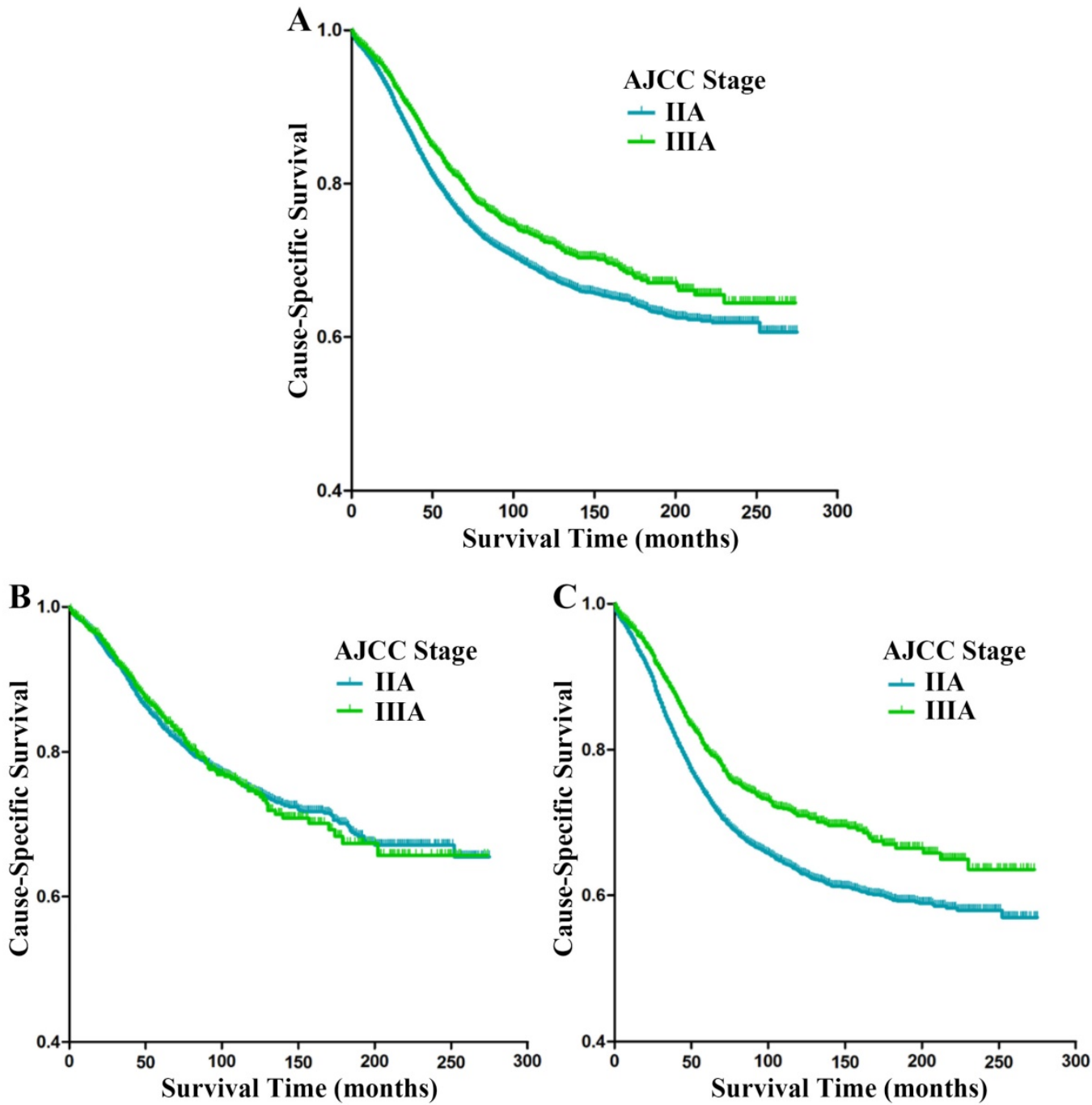

Fig. 1. Cause-specific survival (CSS) stratified by stage IIA and IIIA rectal cancer (A-C) in SEER database. (A) Kaplan-Meier curves for stage IIA and stage IIIA rectal cancer patients. (B) Kaplan-Meier curves for stage IIA and stage IIIA rectal cancer patients with at least 12 lymph nodes harvested. (C) Kaplan-Meier curves for stage IIA and stage IIIA rectal cancer patients with fewer than 12 lymph nodes harvested.

stage IIIA rectal cancer were $83.1 \%$ and $84.8 \%$, respectively. In $\mathrm{LNH}<12$ subgroup, the Kaplan-Meier analysis found stage IIA patients had worse CSS compared with stage IIIA patients ( $p<0.001$, Fig. $1 C)$; the 5-year CSS of patients with stage IIA and stage IIIA rectal cancer were $72.5 \%$ and $79.5 \%$, respectively. Univariate analysis of the subgroup of patients with $\mathrm{LNH}<12$ suggested the following risk factors for CSS: sex $(p=0.088)$, age at diagnosis $(p<0.001)$, race $(p<0.001)$, histological type $(p<0.001)$, pathology grade $(p<0.001)$ and tumor stage $(p<0.001)$. Multivariate Cox proportional model analysis identified sex $(p=0.023)$, age at diagnosis $(p<0.001)$, race $(p<0.001)$, histological type $(p<0.001)$, tumor size $(p<0.001)$, pathological grade $(p<0.001)$, and tumor stage $(p<0.001)$ as independent prognostic factors (Table 4). Compared with stage IIA patients, stage IIIA patients had better CSS (HR 0.805, 95\% CI 0.719-0.901, $\mathrm{p}<0.001$ ).
Further analysis was conducted in subgroups of stage IIIA (T1N1, T2N1 and T1N2a) and stage IIA (T3N0) rectal cancer patients. Kaplan-Meier analysis revealed significant differences between patients with T3N0, T1N1, T2N1 and T1N2a rectal cancer $(p<0.001$, Fig. 2A); the 5-year CSS for patients with T3N0, T1N1, $\mathrm{T} 2 \mathrm{~N} 1$ and $\mathrm{T} 1 \mathrm{~N} 2 \mathrm{a}$ rectal cancer were $77.1 \%, 85.9 \%$, $80.0 \%$ and $80.6 \%$, respectively. Multivariate analysis identified some independent prognostic factors presented following: sex $(\mathrm{p}<0.001)$, age at diagnosis $(p<0.001)$, race $(p<0.001)$, histological type $(p<0.001)$, tumor size $(p<0.001)$, pathological grade $(p<0.001)$, LNH $(p<0.001)$, and TN category $(p=0.013)$ (Table 3$)$. Compared with T3N0 patients, T1N1 patients showed a higher tendency for better CSS (HR 0.725, 95\% CI 0.588-0.893, $p=0.003$, Table 3), the HR for T2N1 patients was 0.930 (95\% CI 0.842-1.028, $\mathrm{p}=0.155)$, and the HR for T1N2a patients was $1.092(95 \%$ CI $0.685-1.739, \mathrm{p}=0.712)$. 
Table 2. Univariate survival analyses of patients with stage IIA and stage IIIA rectal cancer in SEER database

\begin{tabular}{|c|c|c|c|c|}
\hline Variable & No. & 5-year CSS & $\log \operatorname{rank} \chi^{2}$ & P-value \\
\hline Sex & & & 7.761 & 0.005 \\
\hline Male & 9444 & $77.4 \%$ & & \\
\hline Female & 7344 & $78.7 \%$ & & \\
\hline Age at diagnosis (yr) & & & 359.508 & $<0.001$ \\
\hline$\leq 60$ & 5472 & $86.8 \%$ & & \\
\hline$>60$ & 11316 & $73.1 \%$ & & \\
\hline Race & & & 62.960 & $<0.001$ \\
\hline White & 14005 & $78.0 \%$ & & \\
\hline Black & 1251 & $70.6 \%$ & & \\
\hline Other a & 1532 & $83.1 \%$ & & \\
\hline Histological Type & & & 45.078 & $<0.001$ \\
\hline Adenocarcinoma & 15587 & $78.4 \%$ & & \\
\hline Mucinous adenocarcinoma & 1149 & $72.5 \%$ & & \\
\hline Signet-ring cell carcinoma & 52 & $47.1 \%$ & & \\
\hline Pathology grade & & & 22.805 & $<0.001$ \\
\hline Well & 1280 & $80.5 \%$ & & \\
\hline Moderate & 13345 & $78.7 \%$ & & \\
\hline Poor & 2084 & $73.6 \%$ & & \\
\hline Undifferentiated & 79 & $72.2 \%$ & & \\
\hline LNH & & & 169.261 & $<0.001$ \\
\hline$<12$ & 9230 & $74.0 \%$ & & \\
\hline$\geq 12$ & 7558 & $83.4 \%$ & & \\
\hline Tumor stage & & & 26.426 & $<0.001$ \\
\hline IIA & 13551 & $77.1 \%$ & & \\
\hline IIIA & 3237 & $81.6 \%$ & & \\
\hline TN category & & & 39.339 & $<0.001$ \\
\hline T3N0 & 13551 & $81.6 \%$ & & \\
\hline T1N1 & 850 & $85.9 \%$ & & \\
\hline $\mathrm{T} 2 \mathrm{~N} 1$ & 2285 & $80.0 \%$ & & \\
\hline T1N2a & 102 & $80.6 \%$ & & \\
\hline
\end{tabular}

Table 3. Multivariate survival analyses of patients with stage IIA and stage IIIA rectal cancer in SEER database

\begin{tabular}{|c|c|c|c|c|}
\hline \multirow[t]{2}{*}{ Variables } & \multicolumn{2}{|c|}{ Multivariate analysis } & \multicolumn{2}{|c|}{ Multivariate analysis } \\
\hline & $\mathrm{HR}(95 \% \mathrm{CI})$ & P-value & $\mathrm{HR}(95 \% \mathrm{CI})$ & P-value \\
\hline Sex & & $<0.001$ & & $<0.001$ \\
\hline Male & reference & & reference & \\
\hline Female & $0.882(0.824-0.943)$ & & $0.882(0.825-0.944)$ & \\
\hline Age at diagnosis (yr) & & $<0.001$ & & $<0.001$ \\
\hline$\leq 60$ & reference & & reference & \\
\hline$>60$ & $2.003(1.848-2.172)$ & & $2.002(1.846-2.170)$ & \\
\hline Race & & $<0.001$ & & $<0.001$ \\
\hline White & reference & & reference & \\
\hline Black & 1.537(1.369-1.725) & $<0.001$ & $1.537(1.370-1.726)$ & $<0.001$ \\
\hline Other a & $0.752(0.660-0.856)$ & $<0.001$ & $0.752(0.661-0.856)$ & $<0.001$ \\
\hline Histological Type & & $<0.001$ & & $<0.001$ \\
\hline Adenocarcinoma & reference & & reference & \\
\hline Mucinous adenocarcinoma & $1.154(1.016-1.310)$ & 0.027 & $1.153(1.015-1.309)$ & 0.028 \\
\hline Signet-ring cell carcinoma & $2.524(1.581-4.030)$ & $<0.001$ & $2.580(1.614-4.124)$ & $<0.001$ \\
\hline Tumor size $(\mathrm{cm})$ & $1.019(1.011-1.027)$ & $<0.001$ & $1.018(1.010-1.027)$ & $<0.001$ \\
\hline Pathology grade & & $<0.001$ & & $<0.001$ \\
\hline Well & reference & & reference & \\
\hline Moderate & $1.039(0.913-1.182)$ & 0.565 & $1.033(0.908-1.176)$ & 0.619 \\
\hline Poor & $1.266(1.088-1.473)$ & 0.002 & $1.256(1.079-1.462)$ & 0.003 \\
\hline Undifferentiated & $1.768(1.132-2.761)$ & 0.012 & $1.745(1.118-2.726)$ & 0.014 \\
\hline LNH & & $<0.001$ & & $<0.001$ \\
\hline$<12$ & reference & & reference & \\
\hline$\geq 12$ & $0.711(0.662-0.765)$ & & $0.710(0.661-0.763)$ & \\
\hline Tumor stage & & 0.016 & & / \\
\hline IIA & reference & & / & \\
\hline IIIA & $0.894(0.816-0.979)$ & & / & \\
\hline TN category & & / & & 0.013 \\
\hline T3N0 & / & & reference & \\
\hline T1N1 & / & & $0.725(0.588-0.893)$ & 0.003 \\
\hline $\mathrm{T} 2 \mathrm{~N} 1$ & / & & $0.930(0.842-1.028)$ & 0.155 \\
\hline T1N2a & / & & $1.092(0.685-1.739)$ & 0.712 \\
\hline
\end{tabular}

$\mathrm{HR}=$ hazard ratio, $\mathrm{CI}=$ confidence interval, $\mathrm{LNH}=$ number of lymph nodes harvested

a Includes Native American, Asian, Pacific Islander and Unknown
Table 4. Multivariate survival analyses of stage IIA and stage IIIA rectal cancer patients with fewer than 12 lymph nodes harvested in SEER database

\begin{tabular}{|c|c|c|c|c|}
\hline \multirow[t]{2}{*}{ Variables } & \multicolumn{2}{|c|}{ Multivariate analysis } & \multicolumn{2}{|c|}{ Multivariate analysis } \\
\hline & $\mathrm{HR}(95 \% \mathrm{CI})$ & P-value & $\mathrm{HR}(95 \% \mathrm{CI})$ & P-value \\
\hline Sex & & 0.023 & & 0.024 \\
\hline Male & reference & & reference & \\
\hline Female & $0.909(0.837-0.987)$ & & $0.910(0.838-0.988)$ & \\
\hline Age at diagnosis (yr) & & $<0.001$ & & $<0.001$ \\
\hline$\leq 60$ & reference & & reference & \\
\hline$>60$ & $1.962(1.769-2.176)$ & & $1.961(1.768-2.174)$ & \\
\hline Race & & $<0.001$ & & $<0.001$ \\
\hline White & reference & & reference & \\
\hline Black & $1.448(1.249-1.679)$ & $<0.001$ & $1.450(1.251-1.681)$ & $<0.001$ \\
\hline Other a & $0.788(0.673-0.923)$ & 0.003 & $0.788(0.673-0.923)$ & 0.003 \\
\hline Histological Type & & $<0.001$ & & $<0.001$ \\
\hline Adenocarcinoma & reference & & reference & \\
\hline $\begin{array}{l}\text { Mucinous } \\
\text { adenocarcinoma }\end{array}$ & $1.097(0.936-1.286)$ & 0.251 & $1.095(0.934-1.283)$ & 0.263 \\
\hline Signet-ring cell carcinoma & $2.801(1.725-4.547)$ & $<0.001$ & $2.823(1.735-4.592)$ & $<0.001$ \\
\hline Tumor size $(\mathrm{cm})$ & $1.019(1.010-1.028)$ & $<0.001$ & $1.019(1.010-1.028)$ & $<0.001$ \\
\hline Pathology grade & & $<0.001$ & & $<0.001$ \\
\hline Well & reference & & reference & \\
\hline Moderate & $1.078(0.920-1.264)$ & 0.352 & $1.073(0.915-1.258)$ & 0.386 \\
\hline Poor & $1.391(1.157-1.673)$ & $<0.001$ & $1.377(1.144-1.656)$ & 0.001 \\
\hline Undifferentiated & $1.882(1.126-3.145)$ & 0.016 & $1.848(1.106-3.090)$ & 0.019 \\
\hline Tumor stage & & $<0.001$ & & / \\
\hline IIA & reference & & / & \\
\hline IIIA & $0.805(0.719-0.901)$ & & / & \\
\hline TN category & & / & & 0.001 \\
\hline T3N0 & / & & reference & \\
\hline T1N1 & / & & $0.679(0.532-0.868)$ & 0.002 \\
\hline $\mathrm{T} 2 \mathrm{~N} 1$ & / & & $0.831(0.733-0.941)$ & 0.004 \\
\hline T1N2a & / & & $1.104(0.637-1.915)$ & 0.724 \\
\hline
\end{tabular}

$\mathrm{HR}=$ hazard ratio, $\mathrm{CI}=$ confidence interval

a Includes Native American, Asian, Pacific Islander and Unknown

In the subgroup of patients with $\mathrm{LNH} \geq 12$, Kaplan-Meier analysis showed significant differences between patients with T3N0, T1N1, T2N1 and T1N2a rectal cancer ( $p=0.013$, Fig. $2 B$ ); the 5-year CSS for patients with T3N0, T1N1, T2N1 and T1N2a rectal cancer were $83.1 \%, 91.1 \%, 82.6 \%$ and $87.2 \%$, respectively. However, multivariate analysis suggested that TN category was not an independent prognostic factor for CSS $(p=0.313)$. Compared with T3N0 patients, the HR for T1N1 patients was 0.878 (95\% CI 0.588-1.312, $\mathrm{p}=0.526$ ), that for T2N1 patients was 1.156 (95\% CI 0.980-1.364, $\mathrm{p}=0.086)$, and 0.969 (95\% CI $0.401-2.339, \mathrm{p}=0.944)$ for T1N2a patients. In the subgroup of patients with $\mathrm{LNH}<12$, Kaplan-Meier analysis revealed significant differences between patients with T3N0, T1N1, T2N1 and T1N2a rectal cancer ( $p<0.001$, Fig. 2 C); the 5-year CSS for patients with T3N0, T1N1, T2N1 and T1N2a rectal cancer were $72.5 \%, 83.0 \%, 78.3 \%$ and $73.7 \%$, respectively. Multivariate Cox proportional model confirmed sex $(p=0.024)$, age at diagnosis $(p<0.001)$, race $(p<0.001)$, histological type $(p<0.001)$, tumor size $(p<0.001)$, pathological grade $(p<0.001)$, and $T N$ category $(p=0.001)$ as independent prognostic factors (Table 4). Compared with T3N0 patients, T1N1 patients were more apt to have better CSS (HR 0.679, $95 \%$ CI $0.532-0.868, p=0.002)$, and so were the $\mathrm{T} 2 \mathrm{~N} 1$ 
patients (HR 0.831, 95\% CI 0.733-0.941, $\mathrm{p}=0.004$ ); The HR for T1N2a patients was 1.104 (95\% CI 0.637-1.915, $\mathrm{p}=0.724$ ).

\section{Evaluating the SEER database outcomes in the FUSCC cohort}

Of 585 eligible patients (482 with stage IIA and 103 with stage IIIA) identified in FUSCC, 32 (5.5\%) were documented dying from rectal cancer. The median follow-up time was 55 months (interquartile range, 23-91 months). Patient demographics and pathologic features in FUSCC cohort based on stage IIA and stage IIIA rectal cancer are summarized in Table 1.

The Kaplan-Meier analysis showed that stage IIA patients had a poorer CSS compared with stage
IIIA patients ( $p=0.019$, Fig. 3A); the 5-year CSS of patients with stage IIA and stage IIIA rectal cancer were $77.1 \%$ and $89.2 \%$, respectively. In univariate analysis, factors germane to CSS were age at diagnosis, $\mathrm{LNH}$, and tumor stage $(\mathrm{p}<0.05)($ Table 5$)$. In multivariate analysis, LNH $(p=0.002)$ and tumor stage $(p=0.019)$ were crucial predictors of poorer CSS (Table 5). Subgroup analyses stratified by LNH number were then carried out. Specifically, in the subgroup of patients with $\mathrm{LNH}<12$, the prognosis of stage IIIA patients was markedly better than that of stage IIA patients ( $p=0.019$, Fig. $3 C$ ), while there was no significant difference between these two stages in patients with $\mathrm{LNH} \geq 12$ ( $p=0.180$, Fig. 3B).
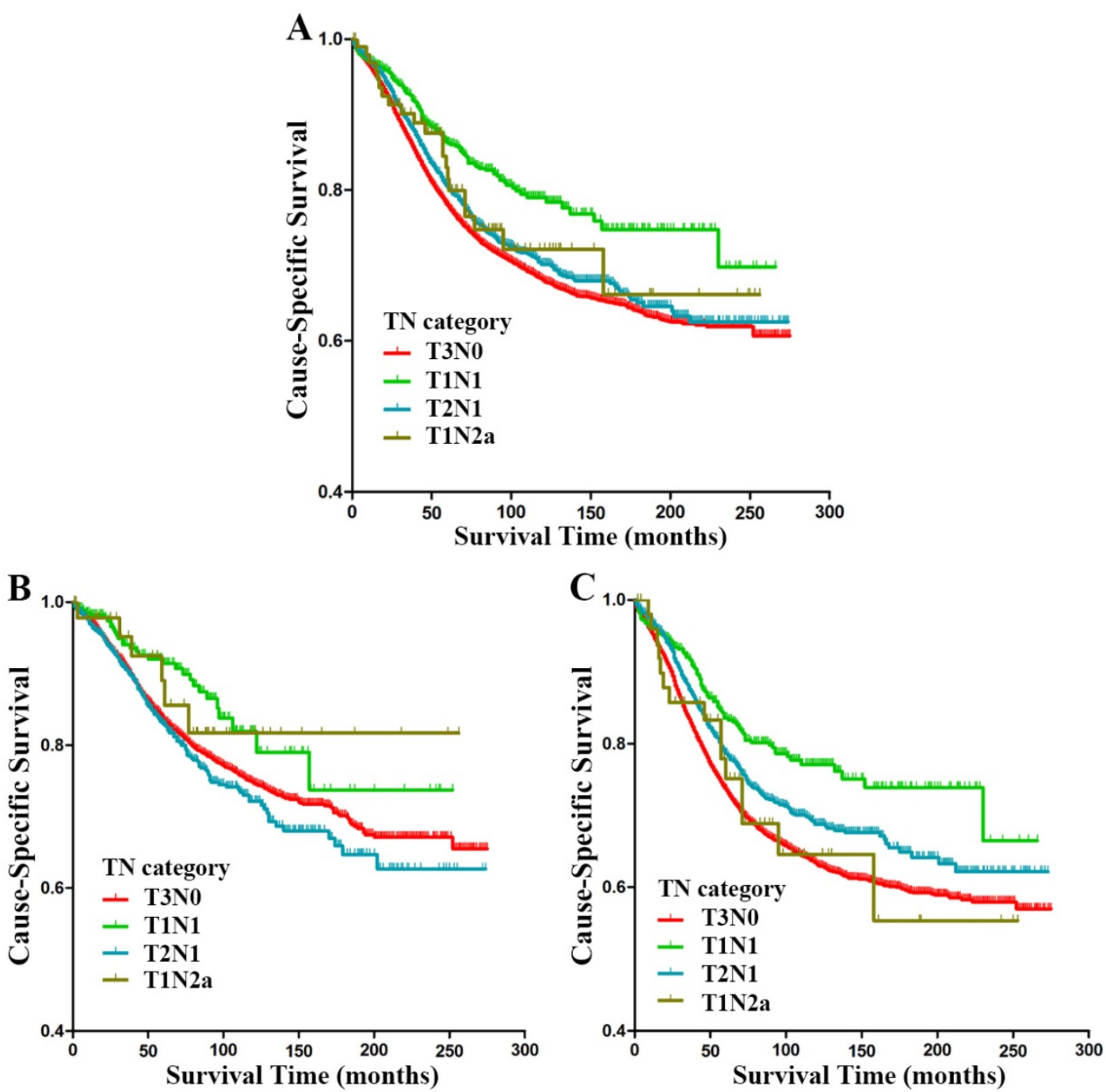

Fig. 2. Cause-specific survival (CSS) stratified by subgroups of stage IIIA (TINI, T2NI and TIN2a) and stage IIA (A-C) in SEER database. (A) Kaplan-Meier curves for T3N0, TIN1, T2N1 and TIN2a rectal cancer patients. (B) Kaplan-Meier curves for T3N0, T1N1, T2N1 and T1N2a rectal cancer patients with at least 12 lymph nodes harvested. (C) Kaplan-Meier curves for T3NO, TINI, T2N1 and TIN2a rectal cancer patients with fewer than 12 lymph nodes harvested. 

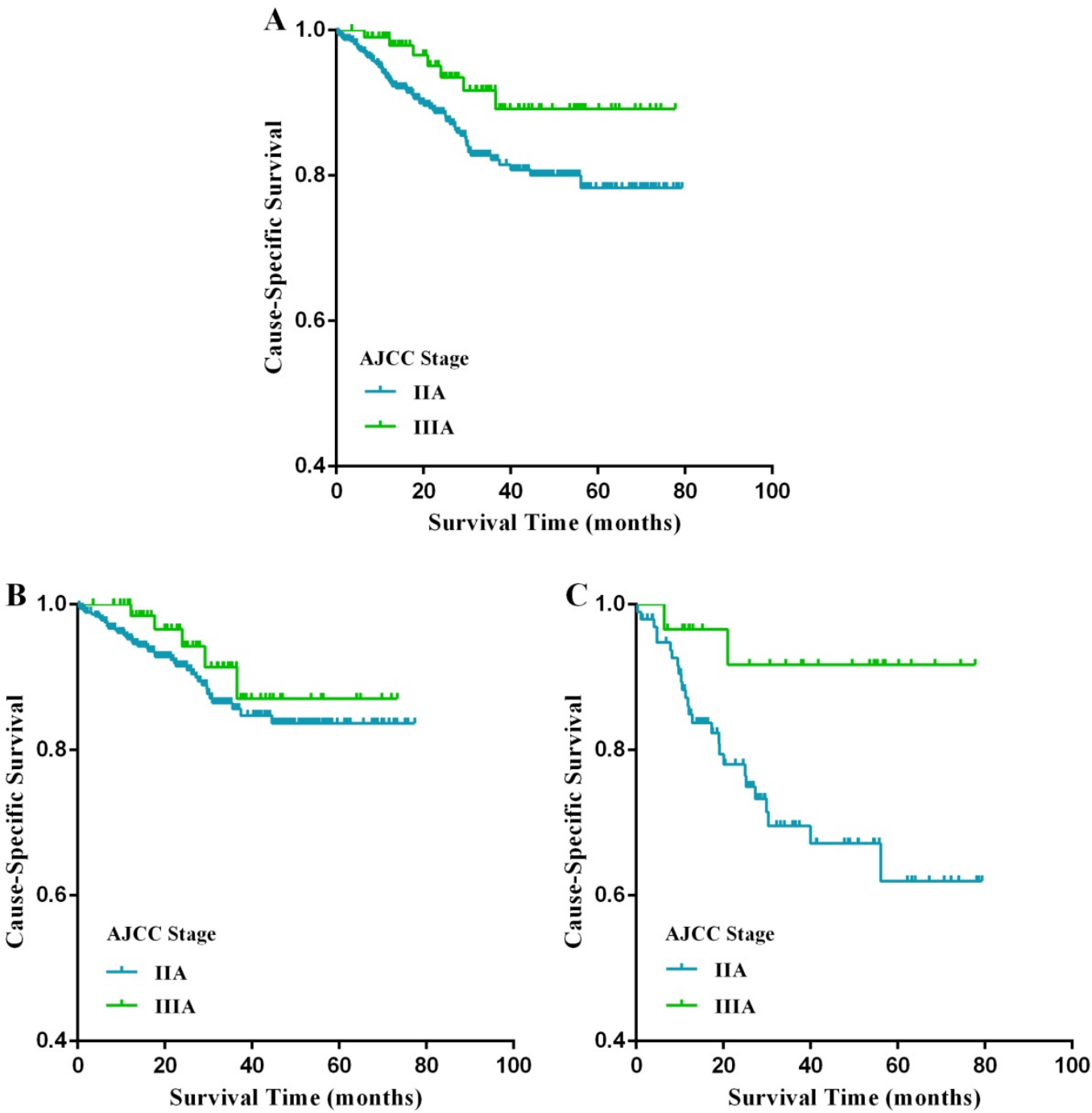

Fig. 3. Cause-specific survival (CSS) stratified by stage IIA and IIIA rectal cancer (A-C) in the FUSCC cohort. (A) Kaplan-Meier curves for stage IIA and stage IIIA rectal cancer patients. (B) Kaplan-Meier curves for stage IIA and stage IIIA rectal cancer patients with at least 12 lymph nodes harvested. (C) Kaplan-Meier curves for stage IIA and stage IIIA rectal cancer patients with fewer than 12 lymph nodes harvested.

Table 5. Univariate and multivariate survival analyses of stage IIA and stage IIIA rectal cancer patients in FUSCC database

\begin{tabular}{|c|c|c|c|c|c|}
\hline \multirow[t]{2}{*}{ Characteristics } & \multicolumn{2}{|r|}{ Univariate analysis } & \multicolumn{2}{|r|}{ Multivariate analysis } & \multirow[b]{2}{*}{ P-value } \\
\hline & 5-year CSS & Log rank $\chi^{2}$ & P-value & $\mathrm{HR}(95 \% \mathrm{Cl})$ & \\
\hline Sex & & 0.603 & 0.437 & & NI \\
\hline Male & $78.2 \%$ & & & & \\
\hline Female & $81.4 \%$ & & & & \\
\hline Age at diagnosis (yr) & & 4.160 & 0.041 & & 0.083 \\
\hline$\leq 60$ & $84.1 \%$ & & & reference & \\
\hline$>60$ & $70.9 \%$ & & & $1.481(0.950-2.309)$ & \\
\hline Histological Type & & 3.030 & 0.082 & & NI \\
\hline Adenocarcinoma & $77.9 \%$ & & & & \\
\hline Mucinous adenocarcinoma & / & & & & \\
\hline Signet-ring cell carcinoma & / & & & & \\
\hline Mean tumor size $(\mathrm{cm})$ & $79.4 \%$ & 2.122 & 0.145 & & NI \\
\hline Pathology grade & & 1.702 & 0.637 & & NI \\
\hline Well & $80.9 \%$ & & & & \\
\hline Moderate & $80.0 \%$ & & & & \\
\hline Poor & $59.7 \%$ & & & & \\
\hline Undifferentiated & / & & & & \\
\hline LNH & & 8.670 & 0.003 & & 0.002 \\
\hline$<12$ & $69.0 \%$ & & & reference & \\
\hline$\geq 12$ & $83.1 \%$ & & & $0.483(0.305-0.765)$ & \\
\hline Tumor stage & & 5.151 & 0.023 & & 0.019 \\
\hline IIA & $77.1 \%$ & & & reference & \\
\hline IIIA & $89.2 \%$ & & & $0.407(0.187-0.885)$ & \\
\hline
\end{tabular}




\section{Discussion}

The $7^{\text {th }}$ edition AJCC cancer staging system defined stage IIIA colorectal cancer as tumors with early stage of intestinal wall invasion and regional lymph node involvement, including T1-2N1M0 and T1N2aM0 lesions[3]. The conflicting oncological outlook of stage IIIA colorectal cancer may be caused by the following features: limited depth of invasion (T1 or T2) and early metastasis to regional lymph node. On the one hand, primary lesions in early $\mathrm{T}$ stages exhibit a relatively shallow depth of invasion, and positive lymph nodes may be confined to the primary lesions area, which can be radically resected without difficulty, giving forth to a pleasant prognosis. However, on the other hand, regional lymph nodes metastasis and early stage primary lesions may present the aggressive biological behavior of these tumors. Therefore, these patients may be associated with poor prognoses. A survival paradox between stage IIIA and stage II colorectal cancer was reported. $\mathrm{Li}$ et al.[4] reorganized the TNM staging system and suggested that stage IIIA should be classified as stage I or stage II based on cluster analysis of the TN scores in colorectal cancer.

The current study indicated that patients with stage IIA rectal cancer had poorer CSS than those with stage IIIA disease, especially when inadequate lymph nodes were examined. However, when adequate lymph nodes were examined, patients with stage IIA rectal cancer had a CSS comparable with patients with stage IIIA lesions. In the subgroup of patients with stage IIIA rectal cancer, T1N1 patients had higher CSS than T2N1 patients and T1N2a patients. When inadequate lymph nodes are harvested, patients with stage IIA (T3N0) rectal cancer had poorer CSS than patients with T1N1 and T2N1 disease. When adequate lymph nodes are harvested, patients with stage IIA (T3N0) rectal cancer had a CSS comparable with patients with T1N1 and T2N1 lesions. We drew the conclusion above from the SEER database. It's not the practice of tertiary care centers alone, but a reflection of general practice. Then the findings were verified by using the data from the FUSCC cohort.

Over-estimation of the relative weighting of the node metastases ( $\mathrm{N}$ stage) in colorectal cancer is the apparent deficiency of the AJCC cancer staging system $[19,20]$. All colorectal cancer with lymph node metastases are defined as stage III disease except for those with distant metastases. Interactions between TN categories upon incorporation into a staging system are complex. For example, the current TNM staging system, in which $\mathrm{N}$ stage has greater weight than local invasion ( $\mathrm{T}$ stage), causes poor monotonicity of gradients from the early tumor stages to the advanced tumor stages[4, 21]. The survival contradiction between stage IIA and stage IIIA rectal cancer indicates the weight of the $\mathrm{N}$ stage may be over-estimated and $\mathrm{T}$ stage is underestimated simultaneously. This conventional concept should be reconsidered and correlated with current survival data of colorectal cancer. Li et al.[4] investigated the relative influences of $\mathrm{T}$ and $\mathrm{N}$ stages on survival outcomes in non-metastatic colorectal cancer through multiple linear regressions, and found that $\mathrm{T}$ stage had greater impacts than $\mathrm{N}$ stage, especially on rectal cancer rather than colon cancer. This may help explain the comparable CSS between patients with stage IIA and stage IIIA rectal cancer when adequate lymph nodes are examined.

In our study, the mean tumor size of stage IIA rectal cancer is larger than that of stage IIIA rectal cancer in both SEER cohort and FUSCC cohort (4.9 vs 3.5 and 4.38 vs 3.17 respectively), which is in accordance with findings in a previous study[6]. Therefore, it's conceivable that en bloc resection of the stage IIIA rectal cancer is much easier to achieve than that of the stage IIA disease, which makes it difficult to guarantee the surgical negative margin of the stage IIA rectal cancer. An observational cohort study conducted by Massarweh et al.[22] found that risk-adjusted pathologic margin positivity rate could be a quality indicator in rectal cancer surgery. Moreover, it's ineluctable that proficiency of performing surgery on locally advanced rectal cancer may vary among surgeons from different hospitals and institutes, thereby increasing the positive resection margin rate in IIA rectal cancer. Furthermore, a retrospective analysis revealed that margin positivity was associated with the recurrence of rectal cancer[23]. Consequently, the larger tumor size of the stage IIA rectal cancer can increase its surgical margin positivity, thus escalating the recurrence rate of rectal cancer and jeopardizing the prognosis of rectal cancer patients.

Stage migration refers to the phenomenon of understaging, which occurs when deficient lymph nodes are examined in colorectal cancer patients[24]. The stage migration phenomenon primarily results from two reasons: inadequate lymph node dissection and insufficient lymph nodes harvested[24, 25]. Metastatic lymph nodes in a surgical specimen of colorectal cancer were occasionally missing, and these patients were subsequently falsely diagnosed as "node-negative" due to an insufficient number of lymph nodes examined. Stage IIA rectal cancer patients with inadequate lymph nodes examined $(<12)$ may be understaged and deprived of the essential adjuvant therapy, leading to poor prognosis. Duraker et al.[26] suggested that stage II colorectal 
cancer patients who had 1 to 7 lymph node(s) or 8 to 11 lymph nodes examined had a poorer survival compared with patients with no less than 12 lymph nodes examined $(p=0.006$ or $p=0.037)$. The phenomenon of stage migration may help shed light on why cases with stage IIA rectal cancer exhibited poorer survival compared with those with stage IIIA lesions when insufficient lymph nodes are harvested.

A consensus was presented by the National Institutes of Health (NIH) Consensus Conference in 1990, which recommended postoperative chemoradiotherapy as the standard treatment modality for patients with stage II or stage III rectal cancer after resection[27-29]. Numerous retrospective studies were performed to identify patients who can avert postoperative therapy and pertinent adverse effects. Single-institution retrospective studies indicated that postoperative radiotherapy may not be necessary for some patients with pT3N0 disease[30, 31]. What's more, it's demonstrated by a pooled analysis of five phase III collaborative group trials that pT1-2N1 and pT3N0 patients may have "intermediate" risk of local recurrence[32]. In these patients, the local recurrence rates were $7 \%$ for the pT1-2N1 group and $9 \%$ for the pT3N0 group, whereas the 5-year overall survival (OS) rates were similar between patients receiving adjuvant chemotherapy alone or adjuvant chemoradiotherapy, indicating postoperative chemotherapy and radiotherapy could be avoided in the selection of patients with pT1-2N1 and T3N0 cancers. The analysis also found T3N0 tumors, without radiotherapy treatment, had a disease-free survival (DFS) rate of $69 \%$ and a 5-year actuarial rate of local recurrence of $11 \%$. In addition, postoperative radiotherapy decreases local failure rates, which nonetheless does not have apparent impact on DFS or OS[28, 33, 34]. Considering the morbidity, decrease in life quality and mortality concerning locoregional recurrence of the disease, many practitioners perceive reduction in local recurrence to be a pleasant outcome. Thereby, definitive conclusions are hard to reach when it comes to which kind of patient can avoid adjuvant radiotherapy, and it should be studied prospectively[32]. Hence, postoperative chemoradiotherapy is currently recommended for treatment of pT3-4 and node-positive rectal cancer.

To our knowledge, our study is the first one to compare the survival outcomes between patients with stage IIA and stage IIIA rectal cancer at different levels of lymph nodes examined. Data of 16,788 patients were from the SEER database. Such a great number of samples can establish credibility in the results, and we further validated these relevant issues in the FUSCC set. Hence, our findings have good reliability. However, there are still several limitations to our study. Some important information about patients and disease cannot be obtained from the SEER database, such as intestinal obstruction or penetration, comorbidities, surgical margin status, circumferential resection margin, and adjuvant chemotherapy data. These clinicopathological information may be of great value to our current analysis. However, the relatively small sample size from the FUSCC set, to some extent, may reduce the statistical power. Moreover, rectal cancer patients receiving preoperative chemoradiotherapy were not included in the current study; thus, our conclusions may not be applicable to these patients.

\section{Conclusion}

In conclusion, stage IIA rectal cancer patients had poorer CSS than those with stage IIIA disease. When insufficient lymph nodes were examined $(\mathrm{LNH}<12)$, the former still had lower CSS than the latter. Nevertheless, when adequate lymph nodes were examined $(\mathrm{LNH} \geq 12)$, patients with stage IIA rectal cancer had a similar CSS to that of the patients with stage IIIA lesions.

\section{Acknowledgements}

This study was supported by the National Key R\&D Program of China (No. 2016YFC0905300 and 2016YFC0905301), the Grant of Science and Technology Commission of Shanghai Municipality (No. 16401970502), the Grant of National Natural Science Foundation of China (No. 81572351), the Shanghai Shenkang Program (No. SHDC12014206), the National Science Foundation of China (No. 81702353) and Shanghai Municipal Natural Science Foundation (17ZR1406400). The funders had no role in the study design, data collection and analysis, decision to publish, or preparation of the manuscript.

\section{Author contribution}

All authors contributed significantly to this work. G.-X.C. proposed, designed and revised the article. Q.-G.L. designed, summarized data and revised this article. S.-B.M., W.-X. D., and W.-Q.X. collected articles, summarized data, did statistical work and drafted the manuscript. B.H., Y.-Q.L., and Y.F. collected articles and summarized information in part. All authors reviewed this manuscript and approved the final draft.

\section{Competing Interests}

The authors have declared that no competing interest exists. 


\section{References}

[1] Siegel R, Miller K, Jemal A. Cancer Statistics, 2017. CA Cancer J Clin 2017;67:7-30.

[2] Chen W, Zheng R, Baade P, et al. Cancer statistics in China, 2015. CA Cancer J Clin 2016;66:115-32.

[3] Edge SB, Compton CC. The American Joint Committee on Cancer: the 7th edition of the AJCC cancer staging manual and the future of TNM. Ann Surg Oncol 2010;17:1471-4.

[4] Li J, Guo BC, Sun LR, et al. TNM staging of colorectal cancer should be reconsidered by T stage weighting. World J Gastroenterol 2014;20:5104-12.

[5] Ueno $H$, Mochizuki $H$, Akagi $Y$, et al. Optimal colorectal cancer staging criteria in TNM classification. J Clin Oncol 2012;30:1519-26.

[6] Huang B, Mo S, Zhu L, et al. The survival and clinicopathological differences between patients with stage IIIA and stage II rectal cancer: An analysis of 12,036 patients in the SEER database. Oncotarget 2016;7:79787-79796.

[7] Kim MJ, Jeong SY, Choi SJ, et al. Survival paradox between stage IIB/C (T4N0) and stage IIIA (T1-2N1) colon cancer. Ann Surg Oncol 2015;22:505-12.

[8] Gunderson LL, Jessup JM, Sargent DJ, et al. Revised tumor and node categorization for rectal cancer based on surveillance, epidemiology, and end results and rectal pooled analysis outcomes. J Clin Oncol 2010;28:256-63.

[9] Hari DM, Leung AM, Lee JH, et al. AJCC Cancer Staging Manual 7th edition criteria for colon cancer: do the complex modifications improve prognostic assessment? J Am Coll Surg 2013;217:181-90.

[10] Kotake K, Honjo S, Sugihara K, et al. Number of lymph nodes retrieved is an important determinant of survival of patients with stage II and stage III colorectal cancer. Jpn J Clin Oncol 2012;42:29-35.

[11] Chang GJ, Rodriguez-Bigas MA, Skibber JM, et al. Lymph node evaluation and survival after curative resection of colon cancer: systematic review. J Natl Cancer Inst 2007;99:433-41.

[12] Compton C, Fenoglio-Preiser CM, Pettigrew N, et al. American Joint Committee on Cancer Prognostic Factors Consensus Conference: Colorectal Working Group. Cancer 2000;88:1739-57.

[13] Compton CC, Greene FL. The staging of colorectal cancer: 2004 and beyond. CA Cancer J Clin 2004;54:295-308.

[14] Sobin LH, Greene FL. TNM classification: clarification of number of regional lymph nodes for pNo. Cancer 2001;92:452.

[15] Compton CC, Fielding LP, Burgart LJ, et al. Prognostic factors in colorectal cancer. College of American Pathologists Consensus Statement 1999. Arch Pathol Lab Med 2000;124:979-94.

[16] Peeples C, Shellnut J, Wasvary H, et al. Predictive factors affecting survival in stage II colorectal cancer: is lymph node harvesting relevant? Dis Colon Rectum 2010;53:1517-23.

[17] Tsai HL, Huang CW, Yeh YS, et al. Factors affecting number of lymph nodes harvested and the impact of examining a minimum of 12 lymph nodes in stage I-III colorectal cancer patients: a retrospective single institution cohort study of 1167 consecutive patients. BMC Surg 2016;16:17.

[18] Li Q, Wang C, Li Y, et al. Lymph node status as a prognostic factor after palliative resection of primary tumor for patients with metastatic colorectal cancer. Oncotarget 2017;8:48333-48342.

[19] Mori T. A comparison of the new (planned) TNM classification and Japanese general rule for staging colorectal cancer. Cancer Invest 2010;28:387-92.

[20] $\mathrm{Li} \mathrm{J}, \mathrm{Yi} \mathrm{CH}, \mathrm{Hu} \mathrm{YT}$, et al. TNM Staging of Colorectal Cancer Should be Reconsidered According to Weighting of the T Stage: Verification Based on a 25-Year Follow-Up. Medicine (Baltimore) 2016;95:e2711.

[21] Lan YT, Yang SH, Chang SC, et al. Analysis of the seventh edition of American Joint Committee on colon cancer staging. Int J Colorectal Dis 2012;27:657-63.

[22] Massarweh NN, Hu CY, You YN, et al. Risk-adjusted pathologic margin positivity rate as a quality indicator in rectal cancer surgery. J Clin Oncol 2014;32:2967-74.

[23] MacLaughlin B, Kumar R. Rectal cancer recurrence in relation to pathologic margin. J Clin Oncol 2015;33:e14645-e14645.

[24] Derwinger K, Carlsson G, Gustavsson B. Stage migration in colorectal cancer related to improved lymph node assessment. Eur J Surg Oncol 2007:33:849-53.

[25] Markl B. Stage migration vs immunology: The lymph node count story in colon cancer. World J Gastroenterol 2015;21:12218-33.

[26] Duraker N, Civelek Caynak Z, Hot S. The prognostic value of the number of lymph nodes removed in patients with node-negative colorectal cancer. Int J Surg 2014;12:1324-7.

[27] Prolongation of the disease-free interval in surgically treated rectal carcinoma. N Engl J Med 1985;312:1465-72.

[28] Krook JE, Moertel CG, Gunderson LL, et al. Effective surgical adjuvant therapy for high-risk rectal carcinoma. N Engl J Med 1991;324:709-15.

[29] NIH consensus conference. Adjuvant therapy for patients with colon and rectal cancer. Jama 1990;264:1444-50.

[30] Willett CG, Badizadegan K, Ancukiewicz M, et al. Prognostic factors in stage T3N0 rectal cancer: do all patients require postoperative pelvic irradiation and chemotherapy? Dis Colon Rectum 1999:42:167-73.

[31] Merchant NB, Guillem JG, Paty PB, et al. T3N0 rectal cancer: results following sharp mesorectal excision and no adjuvant therapy. J Gastrointest Surg 1999;3:642-7.

[32] Gunderson LL, Sargent DJ, Tepper JE, et al. Impact of T and N stage and treatment on survival and relapse in adjuvant rectal cancer: a pooled analysis. J Clin Oncol 2004;22:1785-96.
[33] Fisher B, Wolmark N, Rockette H, et al. Postoperative adjuvant chemotherapy or radiation therapy for rectal cancer: results from NSABP protocol R-01. J Natl Cancer Inst 1988;80:21-9.

[34] Wolmark N, Wieand HS, Hyams DM, et al. Randomized trial of postoperative adjuvant chemotherapy with or without radiotherapy for carcinoma of the rectum: National Surgical Adjuvant Breast and Bowel Project Protocol R-02. J Natl Cancer Inst 2000;92:388-96. 